\author{
Christian Rolfo, Giuseppe Bronte, Leonor Leider, \\ Patrick Pauwels, Konstantinos Papadimitriou, \\ Antonio Russo, and Marc Peeters
}

\title{
14.1 VIPoma
}

\subsubsection{Epidemiology}

VIPomas represent $1.4 \%$ of Gastro entero pancreatic (GEP) neuroendocrine tumours (NETs), corresponding to an incidence of $0.01 / 1,000,000$ yearly. About $85 \%$ are pancreatic, and amongst islet cell tumours, they are about $2 \%$ [1]. Rare extrapancreatic types exist including oesophagus, liver and retroperitoneum, but these forms are mainly present in paediatric age and are attributable to neuroblastomas, which produce also VIP. In adults the extrapancreatic types have low malignant phenotype.

In the pancreas VIPoma usually arises as a unique tumour. It may reach great dimensions, $3 \mathrm{~cm}$ as diameter (range of $1-7 \mathrm{~cm}$ ) on the average, but one case report describes also a $20 \mathrm{~cm}$ VIPoma [2]. The main location is (75\%) within the pancreatic body and tail. It is more frequent in female (about $56 \%$ ) and the mean age at diagnosis is 49 years.

The pure secreting VIPoma is rare, because about $75 \%$ of VIPomas produce also pancreatic polypeptide (PP), neurotensin, gastrin and GIP. However, it is nearly

\footnotetext{
C. Rolfo $\bullet$ K. Papadimitriou $\bullet$ M. Peeters $(\varangle)$

Oncology Department and Multidisciplinary Oncology Center of Antwerp (MOCA),

Antwerp University Hospital, Wilrijkstraat 10, Edegem 2650, Belgium

e-mail: Marc.peeters@uza.be

G. Bronte • A. Russo

Section of Medical Oncology, Department of Surgical, Oncological and Stomatological Sciences, University of Palermo, Palermo, Italy

L. Leider

Pathology Institute, Sourasky Medical Center, Tel Aviv, Israel

P. Pauwels

Pathology Department and MOCA, Antwerp University Hospital, Edegem, Belgium

(C) Springer-Verlag Berlin Heidelberg 2015

S. Yalcin, K. Öberg (eds.), Neuroendocrine Tumours:

Diagnosis and Management, DOI 10.1007/978-3-662-45215-8_14
} 
always biologically active [3, 4]. Four to ten percent of VIPomas belong to the MEN syndrome. Wermer syndrome, induced by VIP production, arises in 1-7\% of islet cell tumours.

\subsubsection{Molecular Mechanisms}

To date we can find in the literature 133 case reports and 161 patients grouped in 8 cohorts on VIPoma. Amongst all publications, a small number of them report the investigation about the molecular mechanisms for the development of VIPoma.

In particular, a study investigated benign and malignant NETs for microsatellite loss of heterozygosity ( $\mathrm{LOH}$ ) analysis and fluorescence in situ hybridization (FISH) in order to evaluate the importance of chromosome $3 p$ deletions in the molecular pathogenesis and biological behaviour. Amongst these patients a VIPoma showed $\mathrm{LOH}$ at all informative microsatellite markers. This finding could indicate that the tumour cells initially had a $3 p$ deletion which was followed by duplication of the remaining allele. Such endoreduplication was also reported for colorectal cancer [5, 6]. In a similar case series, chromosome $6 \mathrm{q}$ losses were observed. Some tumour suppressor genes are located in this region of deletion. These genes include "absent in melanoma 1" (AIM1), "cyclin C" (CCNC) and "receptor-type protein-tyrosine phosphatase kappa" (PTPRK). AIM1 and CCNC were mapped to 6q21 and PTPRK to 6q22.2-q22.3. The first one exerts its effects through interactions with the cytoskeleton. The CCNC is upregulated by $1 \alpha, 25$-dihydroxyvitamin D3, inhibits cellular growth and induces apoptosis. The PTPRK gene seems to be involved in the regulation of cell adhesion by dephosphorylation of $\beta$-catenin and $\gamma$-catenin/plakoglobin or cadherins, thereby contributing to the formation and maintenance of intact adherens junctions [7].

Another report of pancreatic NETs including VIPomas evaluated the putative tumour suppressor gene DPC4 located at the chromosome 18q21.1. This gene, also indicated as Smad4, is a member of the highly conserved family of Smad proteins that are involved in the transduction of signals from the transforming growth factor family of cytokines. This study found out no mutations in this gene for VIPomas [8].

A study about the role of sex chromosome in NET development included 1 VIPoma. This analysis showed a loss of chromosome $\mathrm{X}$ in $40 \%$ of female patients whereas loss of chromosome $\mathrm{Y}$ in $36 \%$ of male patients without loss of the $\mathrm{X}$ chromosome. Sex chromosome loss is associated with aggressiveness of pancreatic NETs and may also predict a poorer clinical outcome. This phenomenon could be explained by the role of genes on the $\mathrm{X}$ chromosome in the induction of senescence and control of cell proliferation [9].

BRAF mutations were also investigated in NET, but these genetic alterations were really low (3\%). However, none of the two VIPomas of this case series bore BRAF gene impairment [10].

Recently, in a patient with VIPoma, LOH downregulation was observed by the microarray analysis for the mismatch repair gene $\mathrm{MSH} 2$ in the primary tumour. Besides, in the same case, a strong overexpression of the chemokine CXCR4 gene 
was found in the liver metastases. On the basis of these findings, the authors argued a role for MSH2 gene impairment in carcinogenesis and for CXCR4 dysregulation in metastasis development [11].

\subsubsection{Clinical Manifestations}

In 1958 Verner and Morrison described in two patients a syndrome including diarrhoea, hypokalaemia, hypochlorhydria and metabolic acidosis [12, 13]. For this syndrome an acronym was used: WDHH (watery diarrhoea, hypokalaemia, hypochlorhydria). It was also defined as "pancreatic cholera", but this definition is not proper when it arises from an extrapancreatic origin. The term VIPoma was suggested by the elevated plasma levels of the vasoactive intestinal polypeptide (VIP), which are associated with this kind of tumour. VIP is a 28-amino acid polypeptide which is distributed throughout the body and normally functions as a neurotransmitter. In the gut it regulates the blood flow, smooth muscle activity, pancreatic and intestinal secretions and inhibits gastric acid production. Excessive circulating VIP concentrations induce secretion in all intestinal segments of $\mathrm{Na}+\mathrm{K}+, \mathrm{Cl}-$ and $\mathrm{HCO} 3-$ as well as water, but also gastric acid secretion, bone resorption, glycogenolysis and vasodilation.

The Verner-Morrison syndrome is characterized by sizeable diarrhoea (1-6 l/ die), which is watery and increasing. The diarrhoea associated with VIPoma is characterized by its persistence for 48-72 $\mathrm{h}$ after fasting and by great faecal volumes of 6-8 $1 /$ die. The secretory nature of the diarrhoea is confirmed if it is refractory to fasting. The hydro-electrolytic disequilibrium is a consequence of diarrhoea and includes hypokalaemia, hypomagnesaemia, hypovolaemia and metabolic acidosis. These events induce lethargy, muscular weakness, cardiac conduction disturbances, weight loss, abdominal pain, paralytic ileus, dyspepsia and gallbladder hypotonia. Flushing could be found in about $20 \%$ of VIPomas, and rarely acute kidney tubular necrosis was reported. In $75 \%$ of VIPoma patients, hypercalcaemia was described, and hypophosphataemia could be associated because of hyperparathyroidism. Hypercalcaemia could be a consequence of paraneoplastic hormonal secretion. The development of hypercalcaemia was attributed to the release of PTHrp by tumour cells. Half of VIPoma patients develop hyperglycaemia because of reduced glucose tolerance, and an increased hepatic glycogenolysis has been hypothesized as a possible explanation for this finding. In $40 \%$ of those patients, hypochlorhydria has been reported. Hypochlorhydria is due to the inhibition of gastric acid production by VIP. Hyperchloremic acidosis can also develop because of low bicarbonate levels as a consequence of severe intestinal loss [14].

In earlier stages the Verner-Morrison syndrome could be confused with diarrhoeas from different origin, including those related with bacterial, viral and parasitic infections and with inflammatory bowel diseases such as ulcerative colitis and Crohn's disease. Since also other neuroendocrine tumours could induce diarrhoea, the one that is related to VIPoma needs to be distinguished. VIP plasma levels allow to recognize those patients affected by this NET. However, VIP could be present in 
various molecular forms, and its levels could exceed $170 \mathrm{pg} / \mathrm{ml}$. These high VIP levels are often associated with high plasma levels of other diarrhoea-inducing molecules, such as pancreatic polypeptide (PP), gastric inhibiting polypeptide (GIP) and prostaglandin E2 (PGE2) [15]. For this reason the already-known role of VIP as the main inducer of diarrhoea has been limited. Besides some VIPoma patients without diarrhoea showed high VIP plasma levels.

Of malignant VIPomas clinical onset is usually accompanied by advanced stage of neoplastic disease. In these patients the clinical management of hydro-electrolytic disequilibrium needs to precede any other treatment. Since the past decades, the use of prednisone has been revealed as a quite efficacious way to manage diarrhoea. Anyway this symptom could be properly contrasted by the treatment with antidiarrhoeal drugs, prostaglandin inhibitors and indole derivatives, since early phases. The treatment with octreotide, a somatostatin analogue, achieved the best control of diarrhoea with subsequent improvement of hydro-electrolytic disequilibrium. In some patients in whom octreotide was delivered for diarrhoea control, a benefit in metastases regression was also obtained [16]. These results have been subsequently implemented by the findings from the PROMID trial about the antitumour benefit on efficacy end point by octreotide in GEP NET patients.

\subsubsection{Biochemical Indicators}

VIPomas are characterized by hypokalaemia in $100 \%$ of patients and hypochlorhydria in $70 \%$, as a consequence of electrolytic loss by secretory diarrhoea, hypercalcaemia in $40 \%$ and hyperglycaemia in $20 \%$. However, this biochemical changes are not sufficient for diagnosis, because only high VIP plasma levels are really mandatory for VIPoma's diagnosis. Since fasting normal VIP values are ranged between 150 and $170 \mathrm{pg} / \mathrm{ml}$, in VIPoma patients hugely higher levels are found in plasma [14]. The VIP levels are usually found elevated in almost all cases, but it could also be found normal between the episodes of diarrhoea. However, the evaluation of VIP plasma levels needs some particular technical devices to guarantee its accuracy and its right interpretation. In fact VIP is a highly unstable protein which easily undergoes a proteolytic degradation [17].

Anyway high VIP plasma levels could be found in patients affected by liver and kidney failure, myocardial infarction, intestinal ischemia, AIDS and laxative-induced diarrhoea.

Besides VIPomas are able to produce other peptides such as PP, calcitonin, gastrin, neurotensin, gastric inhibitory peptide (GIP), serotonin, glucagon, insulin, somatostatin, growth hormone-releasing hormone and peptide histidine-methionine [18].

\subsubsection{Diagnosis}

When a NET has a dimension less than $1 \mathrm{~cm}, \mathrm{CT}$ scan reaches a sensitivity less than $10 \%$. However, VIPomas are usually found when they are greater than $3 \mathrm{~cm}$, so that CT sensitivity could reach $100 \%$ [19]. The magnetic resonance imaging (MRI) could help differentiating the small pancreatic tumours from the surrounding 
normal pancreatic tissue. This imaging technique has reached a sensitivity of 85 and $100 \%$ specificity [20]. On the basis of the high expression of somatostatin receptors in VIPomas, OctreoScan has been considered as a reliable evaluation for this kind of tumours. Somatostatin receptors are expressed in 80-90 \% of VIPomas.

The histological examination of VIPoma show some molecular patterns, such as solid, acinar or trabecular tissue architecture with scant mitoses. The cells are cytologically bland, with modest amount of cytoplasm and round nuclei without visible nucleoli. Few mitotic figures can be found. However, since 60-80\% of VIPomas are metastatic diagnosis, locoregional lymph nodes and suspicious distant metastases could confirm that those histological features are associated with a malignancy [21].

\subsubsection{Treatment Options}

The suspicion of this neuroendocrine tumour in patients with chronic diarrhoea could help to diagnose early this rare disease. Before any curative or palliative treatment is started, the life-threatening hydro-electrolytic disorders must be corrected. Some patients could require a massive intravenous potassium replacement because a consistent potassium deficit induced by chronic gastrointestinal losses.

More than half of VIPomas are resectable at diagnosis. Curable rate by resection reached $10 \%$ [22]. The localization of VIPomas is relatively easy because $80 \%$ have a single site and range between 1 and $7 \mathrm{~cm}$. Pancreatic forms are usually localized in the pancreatic tail. For this reason in these cases, the surgical treatment is represented by a distal pancreatectomy. When pancreatic lesions could not be identified, the exploration of adrenal glands and sympathetic plexus for extrapancreatic forms is necessary. Cytoreductive surgery includes palliative debulking of the primary tumour. This treatment achieves a tumour bulk reduction, the improvement of hormone-mediated symptoms and the prevention of local and systemic tumour effects. A complete resection of the primary tumour improves the prognosis greatly.

In metastatic patients with low risk, the surgical option could be taken into account for symptoms control, which could be reached in $40 \%$ of patients. In highrisk patients with multiple metastatic sites, a systemic treatment is overriding. However, in these patients a palliative debulking surgery should be considered, since patients' outcomes could be improved.

The medical treatment of VIPoma is mainly directed to symptom control. Somatostatin analogues achieved diarrhoea improvement in $56 \%$ of patients. Besides a VIP plasma level reduction was also observed in $60 \%$ [23]. Octreotide was delivered at doses ranging between 50 and 1,500 mcg/die. The best response rate was $83 \%$ with median duration of 6 months. However, octreotide $250-450 \mathrm{mcg} /$ day induced $60 \%$ of symptom control, $70 \%$ of biochemical changes and 5-10\% of tumour responses. The stabilization of neoplastic disease was documented by CT scan in 30-50\% of patients [24]. Some authors reported a marked decrease in the tumour blood flow in two patients with VIPomas. This finding allowed to hypothesize a direct vasoactive effect of octreotide on the tumour blood supply or an indirect effect of reduced hormonal secretion on blood flow [25]. 
Twenty percent of patients experienced resistance to octreotide and $16 \%$ showed a direct antitumour activity.

After a prolonged treatment with octreotide, tachyphylaxis could be observed. For this reason dose escalation or combination with other agents such as interferonalpha could be considered to overcome resistance. In the next future newer somatostatin analogues will be available, such as pasireotide or SOM-230, with prolonged half-life and higher affinity for somatostatin receptors.

As alternative treatment options both chemotherapy and radiotherapy were tested. The former showed no significant benefits and the latter obtained anecdotal benefit but shortly lasting.

\subsection{PPoma}

PPoma belongs to those non-functioning NETs, which are not associated with a clinical syndrome. This group include also tumours secreting neurotensin or calcitonin. This lack of function may be due to the inability of these peptides in the induction of physiopathological changes and subsequent clinical symptoms. In these tumours the symptoms are usually a consequence of compression, if tumour is greater than $5 \mathrm{~cm}$ or of metastases development. About $65 \%$ of these NETs are malignant [26].

PPoma cells show a differentiation that makes them similar to the gamma cells of the Langerhans islets, which represent $10 \%$ of the pancreatic islet cells and secrete $>90 \%$ of total PP $[27,28]$.

PPoma is characterized by higher plasma levels of pancreatic polypeptide (PP, also reported as growth inhibiting factor, GIF, or human pancreatic polypeptide, $\mathrm{hPP}$ ). This peptide was identified in 1972 by two independent laboratories [29]. Its function has not been yet clarified. However, we know that it weakly inhibits pancreatic enzymes and gastric acid secretion and contrasts gallbladder contraction. PP could also be found in islet cell tumours, like $75 \%$ of VIPomas and $25 \%$ of gastrinoma, in multiple endocrine neoplasias (MENs) and in other intestinal and bronchial tumours.

Pure PPomas are rare. They represent less than $1 \%$ of NETs. Until now the literature reports 24 patients with sporadic pancreatic (non-MEN) and 6 with extrapancreatic PPomas. The age of these patients ranged from 20 to 74 with a mean age of 51. The incidence was similar for females and males. It develops more frequently in the head of pancreas and could reach dimensions greater than $5 \mathrm{~cm}$ because of the lack of specific symptoms. Metastases developed in 8 out of the 24 patients reported, but this evolution seems to be not associated with poor survival [30-43].

PPomas are diagnosed by ultrasound and CT scan. These tumours are mostly found incidentally in patients undergoing diagnostic tests for non-specific 
symptoms. The association of high basal plasma PP levels with highly vascular tumours on CT scan should induce the suspicion for PPoma. Other investigations, including endoscopic ultrasound, somatostatin receptor scintigraphy, PET, transhepatic portal venous sampling, selective angiography with secretin injection or surgical exploration, could help for diagnosis.

Sometimes symptoms and clinical signs could be described such as watery diarrhoea, mild hypergastrinaemia, hypokalaemia, higher basal acid production and peptic ulcer, which help to differentiate it from VIPoma. In some patients skin rash, similar to that observed in patients with glucagonoma, was reported, and it disappears as a consequence of tumour removal. However, high PP plasma levels $(>100 \mathrm{pg} / \mathrm{ml})$ are the most relevant feature [31]. Plasma samples for PP evaluation should be taken in the rested fasting state, since PP values were found increased after a meal and exercise, but also in patients with hypoglycaemia and cholinergic stimulation [28]. It is important to highlight that the finding of high PP levels is not sufficient for PPoma diagnosis. In fact, it was found higher also in other NETs $(25-70 \%)$ and in non-neoplastic patients including chronic pancreatitis, kidney failure, diabetes, hypoglycaemia, duodenal ulcer, alcoholism, advanced age and medications (erythromycin, cisapride, laxative abuse) [17].

A method exists to distinguish the neoplastic from the non-neoplastic production of PP. It consists of a test using atropine. When $1 \mathrm{mg}$ intramuscular atropine is delivered, PP secretion is normally suppressed by the vagal cholinergic regulation [44]. PP produced by a tumour is not inhibited by atropine. However, this mechanism is not absolute, because in some patients with PP-secreting tumours, PP levels could be reduced by atropine. This phenomenon was explained by the presence of PP-secreting benign hyperplasia, which responds to atropine delivery [45]. A stimulation test was also developed using secretin infusion, which induces more significant PP level increase in patients with PPoma than in nonneoplastic patients [46]. However, these dynamic tests have not been yet indicated for clinical practice because a wide case series does not exist to confirm their validity.

To date no histological features have been identified to distinguish PPomas from other pancreatic NETs. In fact they show all the typical neuroendocrine characteristics. Electron microscopy could show secretory granules. Only immunohistochemistry could confirm PP expression by tumour cells. A cut-off of more than $50 \%$ of tumour cells expressing PP was established to classify a tumour PPoma, since this peptide could be also expressed by other islet cell tumours.

Since PPoma is biologically inactive, its diagnosis is usually late, when it has already become malignant and of greater dimensions. For this reason often surgery is not radical for this NET. When dimensions are limited, a careful enucleation with a limited normal tissue margin can be considered for selected patients. Cytoreductive surgery for metastases was proposed, but a clear advantage for patients has not yet been demonstrated. 


\section{References}

1. Hoffman PN, Lasek RJ (1975) The slow component of axonal transport. Identification of major structural polypeptides of the axon and their generality among mammalian neurons. J Cell Biol 66:351-366

2. Stinner B, Rothmund M (1992) Actual surgical strategies for endocrine tumors. Onkologie 15:346-354

3. Norton JA, Cromack DT, Shawker TH et al (1988) Intraoperative ultrasonographic localization of islet cell tumors. A prospective comparison to palpation. Ann Surg 207:160-168

4. Elias E, Polak JM, Bloom SR et al (1972) Pancreatic cholera due to production of gastric inhibitory polypeptide. Lancet 2:791-793

5. Barghorn A, Komminoth P, Bachmann D et al (2001) Deletion at 3p25.3-p23 is frequently encountered in endocrine pancreatic tumours and is associated with metastatic progression. J Pathol 194:451-458

6. Giaretti W (1994) A model of DNA aneuploidization and evolution in colorectal cancer. Lab Invest 71:904-910

7. Barghorn A, Speel EJ, Farspour B et al (2001) Putative tumor suppressor loci at $6 \mathrm{q} 22$ and 6q23-q24 are involved in the malignant progression of sporadic endocrine pancreatic tumors. Am J Pathol 158:1903-1911

8. Bartsch D, Hahn SA, Danichevski KD et al (1999) Mutations of the DPC4/Smad4 gene in neuroendocrine pancreatic tumors. Oncogene 18:2367-2371

9. Missiaglia E, Moore PS, Williamson J et al (2002) Sex chromosome anomalies in pancreatic endocrine tumors. Int J Cancer 98:532-538

10. Tannapfel A, Vomschloss S, Karhoff D et al (2005) BRAF gene mutations are rare events in gastroenteropancreatic neuroendocrine tumors. Am J Clin Pathol 123:256-260

11. Müller S, Kupka S, Königsrainer I et al (2012) MSH2 and CXCR4 involvement in malignant VIPoma. World J Surg Oncol 10:264

12. Verner JV, Morrison AB (1958) Islet cell tumor and a syndrome of refractory watery diarrhea and hypokalemia. Am J Med 25:374-380

13. Verner JV, Morrison AB (1974) Endocrine pancreatic islet disease with diarrhea. Report of a case due to diffuse hyperplasia of nonbeta islet tissue with a review of 54 additional cases. Arch Intern Med 133:492-499

14. Mekhjian HS, O’Dorisio TM (1987) VIPoma syndrome. Semin Oncol 14:282-291

15. Bloom SR, Lee YC, Lacroute JM et al (1983) Two patients with pancreatic apudomas secreting neurotensin and VIP. Gut 24:448-452

16. Clements D, Elias E (1985) Regression of metastatic vipoma with somatostatin analogue SMS 201-995. Lancet 1:874-875

17. Modlin IM, Tang LH (1997) Approaches to the diagnosis of gut neuroendocrine tumors: the last word (today). Gastroenterology 112:583-590

18. Perry RR, Vinik AI (1995) Clinical review 72: diagnosis and management of functioning islet cell tumors. J Clin Endocrinol Metab 80:2273-2278

19. King CM, Reznek RH, Dacie JE, Wass JA (1994) Imaging islet cell tumours. Clin Radiol 49:295-303

20. Thoeni RF, Mueller-Lisse UG, Chan R et al (2000) Detection of small, functional islet cell tumors in the pancreas: selection of MR imaging sequences for optimal sensitivity. Radiology 214:483-490

21. Capella C, Polak JM, Buffa R et al (1983) Morphologic patterns and diagnostic criteria of VIPproducing endocrine tumors. A histologic, histochemical, ultrastructural, and biochemical study of 32 cases. Cancer 52:1860-1874

22. Thompson GB, van Heerden JA, Grant CS et al (1988) Islet cell carcinomas of the pancreas: a twenty-year experience. Surgery 104:1011-1017

23. Debas HT, Gittes G (1993) Somatostatin analogue therapy in functioning neuroendocrine gut tumors. Digestion 54(Suppl 1):68-71 
24. Oberg K (1999) Neuroendocrine gastrointestinal tumors-a condensed overview of diagnosis and treatment. Ann Oncol 10(Suppl 2):S3-S8

25. Cho KJ, Vinik AI (1990) Effect of somatostatin analogue (octreotide) on blood flow to endocrine tumors metastatic to the liver: angiographic evaluation. Radiology 177:549-553

26. Kent RB, van Heerden JA, Weiland LH (1981) Nonfunctioning islet cell tumors. Ann Surg 193:185-190

27. Glaser B, Vinik AI, Sive AA, Floyd JC (1980) Plasma human pancreatic polypeptide responses to administered secretin: effects of surgical vagotomy, cholinergic blockade, and chronic pancreatitis. J Clin Endocrinol Metab 50:1094-1099

28. Greenberg GR, McCloy RF, Adrian TE et al (1978) Inhibition of pancreas and gallbladder by pancreatic polypeptide. Lancet 2:1280-1282

29. Kimmel JR, Pollock HG, Hazelwood RL (1968) Isolation and characterization of chicken insulin. Endocrinology 83:1323-1330

30. Bellows C, Haque S, Jaffe B (1998) Pancreatic polypeptide islet cell tumor: case report and review of the literature. J Gastrointest Surg 2:526-532

31. Choksi UA, Sellin RV, Hickey RC, Samaan NA (1988) An unusual skin rash associated with a pancreatic polypeptide-producing tumor of the pancreas. Ann Intern Med 108:64-65

32. Colović R, Grubor N, Micev M et al (2003) Two cases of pancreatic head polypeptide tumors, one with a central cavity which fistulized into the duodenum. Srp Arh Celok Lek 131:259-265

33. Friesen SR, Kimmel JR, Tomita T (1980) Pancreatic polypeptide as screening marker for pancreatic polypeptide apudomas in multiple endocrinopathies. Am J Surg 139:61-72

34. Hayes MM (1980) Report of a pancreatic polypeptide-producing islet-cell tumour of the pancreas causing the watery diarrhoea, hypokalaemia, achlorhydria syndrome in a 55 year old Zimbabwean African male. Cent Afr J Med 26:195-197

35. Mehring UM, Jäger HJ, Klöppel G, Hasse FM (1997) Pancreatic polypeptide secreting endocrine pancreas tumor associated with multiple stomach and duodenal ulcers. Langenbecks Arch Chir 382:134-137

36. Mortenson M, Bold RJ (2002) Symptomatic pancreatic polypeptide-secreting tumor of the distal pancreas (PPoma). Int J Gastrointest Cancer 32:153-156

37. Nobin A, Berg M, Ericsson M et al (1984) Pancreatic polypeptide-producing tumors. Report on two cases. Cancer 53:2688-2691

38. Quin JD, Marshall DA, Fisher BM, MacCuish AC (1991) Metastatic pancreatic polypeptide producing tumour presenting with diabetes mellitus. Scott Med J 36:143

39. Raffel A, Krausch M, Schulte KM, Röher HD (2004) Symptomatic pure pancreatic polypeptidecontaining tumor of the pancreas. Pancreas 29:83

40. Rossi V, Saibeni S, Sinigaglia L et al (2006) Hypokalemic rhabdomyolysis without watery diarrhea: an unexpected presentation of a pancreatic neuro-endocrine tumor. Am J Gastroenterol 101:669-672

41. Strodel WE, Vinik AI, Lloyd RV et al (1984) Pancreatic polypeptide-producing tumors. Silent lesions of the pancreas? Arch Surg 119:508-514

42. Tomita T, Friesen SR, Kimmel JR (1986) Pancreatic polypeptide-secreting islet cell tumor. A follow-up report. Cancer 57:129-133

43. Fuertes Guiró F, Mortara G, Schiaffino E, d'Urbano C (1994) Pancreatic endocrine F-cell tumor. Rev Esp Enferm Dig 86:694-698

44. Schwartz TW, Holst JJ, Fahrenkrug J et al (1978) Vagal, cholinergic regulation of pancreatic polypeptide secretion. J Clin Invest 61:781-789

45. Schwartz TW (1978) Atropine suppression test for pancreatic polypeptide. Lancet 2:43-44

46. Vinik AI, Moattari AR (1989) Treatment of endocrine tumors of the pancreas. Endocrinol Metab Clin North Am 18:483-518 